\title{
MicroRNA-10b pleiotropically regulates invasion, angiogenicity and apoptosis of tumor cells resembling mesenchymal subtype of glioblastoma multiforme
}

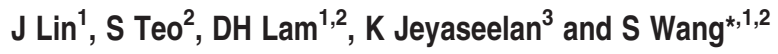

Glioblastoma multiforme (GBM) is a heterogeneous disease despite its seemingly uniform pathology. Deconvolution of The Cancer Genome Atlas's GBM gene expression data has unveiled the existence of distinct gene expression signature underlying discrete GBM subtypes. Recent conflicting findings proposed that microRNA (miRNA)-10b exclusively regulates glioma growth or invasion but not both. We showed that silencing of miRNA-10b by baculoviral decoy vectors in a glioma cell line resembling the mesenchymal subtype of GBM reduces its growth, invasion and angiogenesis while promoting apoptosis in vitro. In an orthotopic human glioma mouse model, inhibition of miRNA-10b diminishes the invasiveness, angiogenicity and growth of the mesenchymal subtype-like glioma cells in the brain and significantly prolonged survival of glioma-bearing mice. We demonstrated that the pleiotropic nature of miRNA-10b was due to its suppression of multiple tumor suppressors, including TP53, FOXO3, CYLD, PAX6, PTCH1, HOXD10 and NOTCH1. In particular, siRNA-mediated knockdown experiments identified TP53, PAX6, NOTCH1 and HOXD10 as invasion regulatory genes in our mesenchymal subtype-like glioma cells. By interrogating the REMBRANDT, we noted that dysregulation of many direct targets of miRNA-10b was associated with significantly poorer patient survival. Thus, our study uncovers a novel role for miRNA-10b in regulating angiogenesis and suggests that miRNA-10b may be a pleiotropic regulator of gliomagenesis.

Cell Death and Disease (2012) 3, e398; doi:10.1038/cddis.2012.134; published online 4 October 2012

Subject Category: Neuroscience

Glioblastoma multiforme (GBM) remains incurable owing to its aggressive growth, invasion and angiogenesis. ${ }^{1}$ The Cancer Genome Atlas (TCGA) Research Network performed a multifaceted molecular and genomic analysis on over 200 GBM specimens, ${ }^{1}$ generating a valuable repository for understanding gliomagenesis. TCGA's data have been deconvoluted to highlight existence of four subtypes existing within a pathologically uniform group of GBM, including mesenchymal, classical, proneural and neural subtypes. ${ }^{2}$ This raises the question of whether an oncogenic microRNA (miRNA) regulates an identical group of genes in all GBM subtypes given the unique gene expression underlying each subtype. $^{2}$

MiRNA-10b has been extensively studied in several pathologies $^{3-6}$ and miR-10b is highly upregulated in human GBM compared with normal brain tissues. ${ }^{3,5}$ However, conflicting evidence has arisen with regards to miR-10b's role in GBM etiology..$^{3,5}$ Gabriely et al. ${ }^{3}$ showed that miR-10b regulates GBM growth and death in vitro and in subcutaneous U87 tumors but does not regulate in vitro GBM cell migration and invasion, while Sun et al. ${ }^{5}$ showed that miR-10b regulates glioblastoma cell invasion but not cell growth in vitro. We speculate that deficiencies in tumor models used in those studies may account for their differing findings. Commonly used GBM lines form well-circumscribed tumors that do not resemble human GBM. Several groups endeavored to improve the orthotopic GBM xenograft model. Notably, CD133-positive 'cancer stem cells' isolated from human $\mathrm{GBMs}^{7}$ and experimental lung metastasis (ELM) assays that enrich for metastatic GBM cells ${ }^{8}$ produce highly invasive and angiogenic orthotopic tumors in mouse models.

Subjecting U87 glioma cells to ELM assay, we established a subline of $U 87$ resembling the mesenchymal subtype of GBM, termed U87-2M1. We used baculoviral vectors, which display high transduction efficiency in glioma cells, ${ }^{9}$ to construct miR$10 \mathrm{~b}$ decoy vectors to study its functions in U87-2M1 cells. We demonstrate that inhibition of miR-10b resulted in distinct increase in apoptosis with a concurrent suppression of glioma cell invasion and angiogenesis in vitro and in vivo. miRNA10b's pleiotropic nature is due to its suppression of multiple tumor suppressors, including FOXO3, CYLD, HOXD10, TP53, PAX6, PTCH1 and NOTCH1. In addition, invasiveness of U87-2M1 cells is partly mediated by miR-10b's suppression of TP53, PAX6, NOTCH1 and HOXD10.

\footnotetext{
${ }^{1}$ Institute of Bioengineering and Nanotechnology, Singapore, Singapore; ${ }^{2}$ Department of Biological Sciences, National University of Singapore, Singapore, Singapore and ${ }^{3}$ Department of Biochemistry, National University of Singapore, Singapore, Singapore

${ }^{*}$ Corresponding author: S Wang, Department of Biological Sciences, National University of Singapore, Singapore 117543, Singapore. Tel: +65 68747712 ; Fax: +65 6779 2486; E-mail: dbsws@nus.edu.sg

Keywords: microRNA-10b; angiogenicity; invasion; apoptosis; glioblastoma

Abbreviations: GBM, glioblastoma multiforme; TCGA, The Cancer Genome Atlas; miRNA, microRNA; ELM, experimental lung metastasis; DAVID, Database for Annotation, Visualization and Integrated Discovery; EMT, epithelial-mesenchymal transition; UTR, untranslated region; Shh, sonic hedgehog; NHA, normal human astrocytes; HUVEC, human umbilical venous endothelial cells; FDR, false discovery rate

Received 8.8.12; accepted 20.8.12; Edited by A Verkhratsky
} 


\section{Results}

U87-2M1 is an invasive subline of U87 glioma cells and resembles the mesenchymal subtype of GBM. Orthotopic xenograft mouse models of GBM utilizing existing human GBM lines poorly mirror the aggressiveness of human GBM. We adopted the ELM assay ${ }^{8}$ to select for invasive U87 GBM cells. U87 GBM cells injected into tail vein of nude mice form lung metastases that were propagated in vitro and named U87-2M1. Orthotopic U87-2M1 tumors in mouse display infiltrative finger-like projections, unlike U87 tumors that

maintain a distinct boundary with brain parenchyma and display little evidence of localized invasion (Figure 1a). U87$2 \mathrm{M} 1$ cells are of metastatic origin but do not metastasize extracranially (unpublished observations), agreeing with published findings that ELM assay-derived glioma cells remain incompetent at metastasizing extracranially ${ }^{8}$ and are behaviorally similar to human GBMs.

To gain an overview of differences in gene expression between U87-2M1 and U87 cells, we performed expression profiling on the Affymetrix whole human genome platform (Affymetrix, Santa Clara, CA, USA). Using gene signatures a

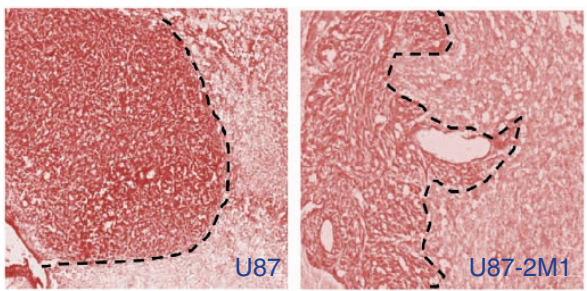

C

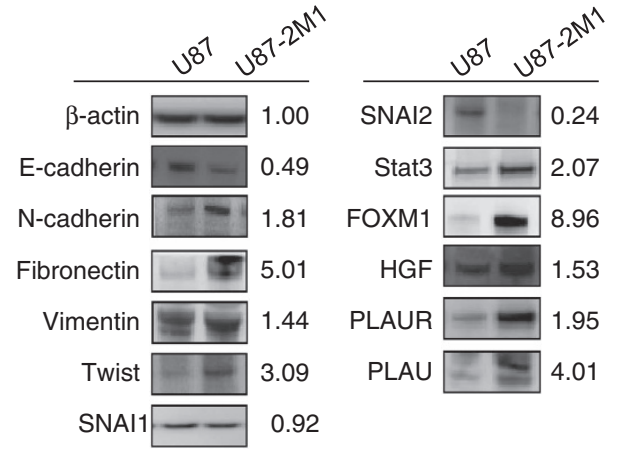

e
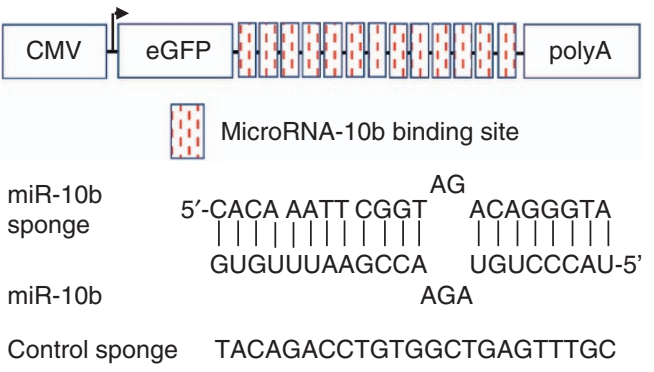

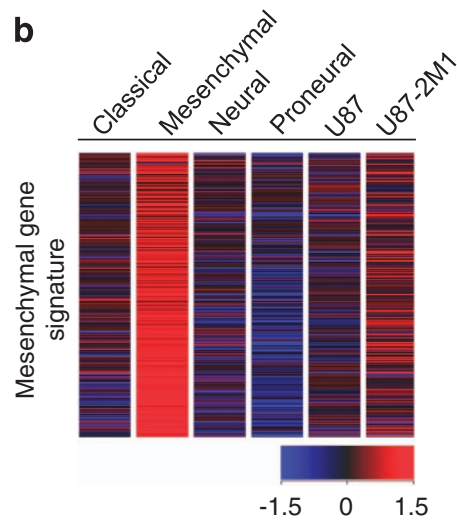

d
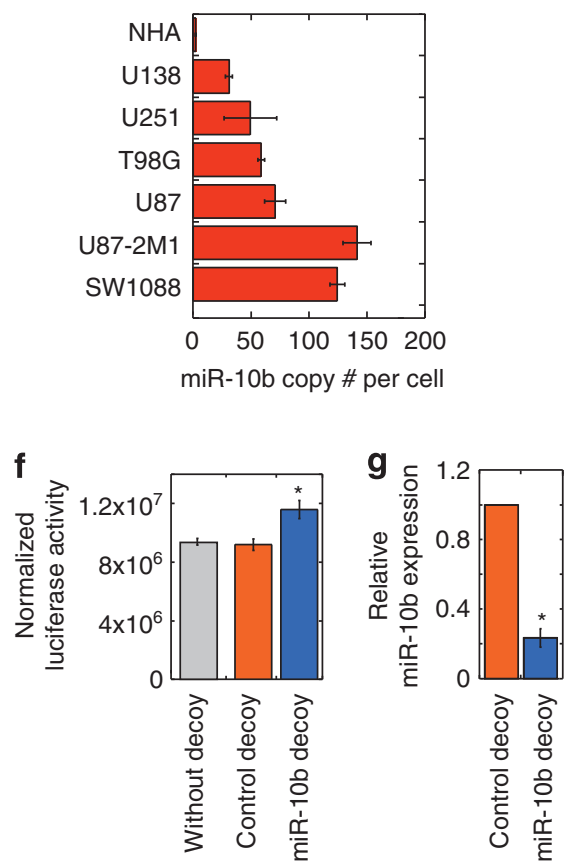

Figure 1 Expression of miR-10b is elevated in invasive mesenchymal subtype-like U87-2M1 glioma cells. (a) Invasive U87-2M1 glioma cell growth in the brain. U87 and U87-2M1 glioma cells were grown intracranially in nude mice for 3 weeks. Tumor growth was visualized by H\&E staining of brain tissues. Bars equal $200 \mu \mathrm{m}$. (b) Heatmap distinctly shows that the mesenchymal gene expression signature is highly activated in the mesenchymal subtype of GBM and U87-2M1 cells, but not in the neural, proneural and classical subtypes, of GBM and U87 glioma cells. (c) Western blotting analysis of expression of invasiveness-associated proteins in U87-2M1 cells as compared with those in U87 cells. Numbers indicate relative protein expression in U87-2M1 compared with U87 cells after normalization to beta-actin levels. Blots shown are representative of biological triplicates. (d) qPCR quantification of miR-10b expression in glioma cell lines (SW1088, U87-2M1, U87, T98G, U251 and U138) and NHA revealed an upregulation of miR-10b expression in glioma cell lines. (e) Construction of control or miR-10b decoy vector. Upper, schematic of the expression cassette. Bottom, sequence of the miR10b-binding site and control sequence with no known complementarity to any known miRNA. (f) miR-10b decoy vector, but not the control decoy vector, relieves the suppression of luciferase expression by endogenous miR-10b in U87-2M1 glioma cells. Error bars represent S.E.from three independent experiments; ${ }^{*} P<0.05$. (g) miR-10b decoy vector decreases the level of detectable miR-10b in U87-2M1 glioma cells. Error bars represent S.E. from three independent transductions; ${ }^{*} P<0.05$ 
representative of the four identified GBM subtypes, ${ }^{2}$ we demonstrate that the gene expression profile of U87-2M1, but not U87 cells, highly resembles the mesenchymal subtype of GBM (Figure 1b, Supplementary Figure S1). In comparison, we were unable to identify any similarity of U87 cells to any subtype of GBM (Figure 1b, Supplementary Figure S1). Genes that were differentially regulated between U87-2M1 and U87 cells were also analyzed using Database for Annotation, Visualization and Integrated Discovery (DAVID) ${ }^{10}$ to identify gene ontologies that were significantly overrepresented. In line with our observation of U87-2M1's invasiveness in vivo, gene ontology terms such as 'cell adhesion', 'vasculature development' and 'extracellular structure organization' came up among the top five most enriched terms (Table 1a). We hypothesize that gene ontology terms 'system development' and 'organ development' hint at the presence of a cancer-associated reprogramming such as an epithelial-mesenchymal transition (EMT). By western blotting, we noted a clear switch from $\mathrm{E}$-cadherin to $\mathrm{N}$-cadherin expression and increased expression of mesenchymal proteins such as vimentin and fibronectin (Figure 1c). Repression of E-cadherin is likely to be mediated by EMT regulator TWIST but not SNAI1 or SNAI2 (Figure 1c). Enhanced expression of STAT3, FOXM1, HGF, PLAUR and PLAU (Figure 1c), which have been previously associated with the mesenchymal, invasive and angiogenic gene expression in gliomas, ${ }^{11-13}$ support U87-2M1's observed invasiveness in vivo.

We asked if miR-10b underlies the enhanced invasiveness of U87-2M1 cells and assayed the copy number of miR-10b in U87-2M1, five other glioma lines and normal human astrocytes (NHA). Consistent with evidence that Twist transcribes miR-10b, ${ }^{4}$ miR-10b copy number in U87-2M1 cells is approximately twice higher than in U87 cells (Figure 1d). U87-2M1 was chosen for further studies to elucidate miR$10 \mathrm{~b}$ 's role in vitro and in vivo.

To investigate miR-10b's role in GBM, we designed a miR$10 \mathrm{~b}$ decoy vector containing 13 repeats of a miR-10b-binding site, and a control vector with no known complementarity to any known miRNA (Figure 1e). Given its high transduction efficiency in glioma cells, ${ }^{9}$ we used baculovirus as delivery vectors. To demonstrate specificity of our miR-10b decoy vector, U87-2M1 cells were transfected with a luciferase vector containing a miR-10b-binding site in its $3^{\prime}$ untranslated region (UTR) and transduced with either baculoviral miR-10b decoy vector or control decoy vector. When the luciferase transgene is co-expressed with a miR-10b-binding site, our miR-10 decoy vector relieved the suppressive effect of endogenous miR-10b on luciferase expression compared with control vector (Figure 1f). We studied endogenous expression of miR-10b after transduction with miR-10b decoy vector and detected a $75 \%$ decrease in miR-10b expression,

Table 1 Characterization of mesenchymal subtype-like U87-2M1 glioma cells by DNA microarray analysis

\begin{tabular}{|c|c|c|c|c|c|}
\hline Gene ontology term & Enrichment score & Count & $\%$ & $P$-value & FDR \\
\hline \multicolumn{6}{|c|}{$\begin{array}{l}\text { (a) Enriched gene clusters in U87-2M1 glioma cells } \\
\text { G0:0007155 }\end{array}$} \\
\hline $\begin{array}{l}\text { Cell adhesion } \\
\text { GO:0048731 }\end{array}$ & 7.16 & 72 & 7.64 & $5.27 E-08$ & $9.52 E-05$ \\
\hline $\begin{array}{l}\text { System development } \\
\text { GO:0001944 }\end{array}$ & 5.33 & 182 & 19.3 & $8.15 \mathrm{E}-09$ & $1.47 \mathrm{E}-05$ \\
\hline $\begin{array}{l}\text { Vasculature development } \\
\text { GO:0043062 }\end{array}$ & 5.12 & 37 & 3.92 & $7.75 \mathrm{E}-08$ & $1.40 E-04$ \\
\hline $\begin{array}{l}\text { Extracellular structure organization } \\
\text { GO:0042060 }\end{array}$ & 3.89 & 30 & 3.18 & $1.11 E-08$ & $2.01 \mathrm{E}-05$ \\
\hline $\begin{array}{l}\text { Wound healing } \\
\text { GO:0048513 }\end{array}$ & 3.46 & 27 & 2.86 & $9.07 \mathrm{E}-06$ & $1.64 \mathrm{E}-02$ \\
\hline Organ development & 3.32 & 132 & 14.0 & $8.21 E-06$ & $1.48 \mathrm{E}-02$ \\
\hline \multicolumn{6}{|c|}{$\begin{array}{l}\text { (b) Enriched gene clusters in U87-2M1 glioma cells after miR-10b silencing } \\
\text { GO:0019222 }\end{array}$} \\
\hline $\begin{array}{l}\text { Regulation of metabolic process } \\
\text { GO:0042127 }\end{array}$ & 6.93 & 401 & 24.4 & $1.36 \mathrm{E}-11$ & $2.53 E-08$ \\
\hline $\begin{array}{l}\text { Regulation of cell proliferation } \\
\text { GO:0048522 }\end{array}$ & 5.26 & 111 & 6.74 & $2.99 \mathrm{E}-08$ & $5.53 E-05$ \\
\hline $\begin{array}{l}\text { Positive regulation of cellular process } \\
\text { GO:0032502 }\end{array}$ & 5.05 & 221 & 13.4 & $6.95 E-09$ & $1.29 \mathrm{E}-05$ \\
\hline GO:0000280 & 4.52 & 333 & 20.2 & $3.42 \mathrm{E}-07$ & $6.34 \mathrm{E}-04$ \\
\hline $\begin{array}{l}\text { Nuclear division } \\
\text { GO:0051270 }\end{array}$ & 3.86 & 39 & 2.37 & $1.19 \mathrm{E}-05$ & $2.21 \mathrm{E}-02$ \\
\hline $\begin{array}{l}\text { Regulation of cell motion } \\
\text { GO:0001944 }\end{array}$ & 3.67 & 35 & 2.13 & $2.22 \mathrm{E}-05$ & $4.10 E-02$ \\
\hline $\begin{array}{l}\text { Vasculature development } \\
\text { GO:0043067 }\end{array}$ & 3.61 & 43 & 2.61 & $9.54 \mathrm{E}-06$ & $1.77 \mathrm{E}-02$ \\
\hline Regulation of programmed cell death & 3.56 & 105 & 6.38 & $4.85 E-06$ & $8.99 E-03$ \\
\hline
\end{tabular}

Abbreviation: FDR, false discovery rate

${ }^{\mathrm{a}}$ Genes that were differentially regulated by more than twofolds between U87-2M1 and U87 cells were screened using DAVID version 6.7, for enriched biological themes. Six most enriched gene ontology terms are listed

${ }^{b}$ Genes that were differentially regulated by more than 1.5-fold after miR-10b silencing in U87-2M1 cells were screened with DAVID version 6.7, for identification of biological themes. Eight most enriched gene ontology terms are listed 
further affirming our decoy vector's specificity in inhibiting miR-10b (Figure 1g).

To demonstrate functional interference of miR-10b in U872M1, we transduced U87-2M1 cells with our control or miR$10 \mathrm{~b}$ decoy vector and performed gene expression profiling. Differentially regulated genes were analyzed using DAVID ${ }^{10}$ to identify enriched gene ontologies. Terms such as 'regulation of cell proliferation', 'nuclear division' and 'regulation of programmed cell death' were significantly overrepresented (Table 1b), supporting prior evidence of a regulatory relationship between $\mathrm{miR}-10 \mathrm{~b}$ and cell growth. ${ }^{3}$ Strikingly, enrichment of gene ontologies representing 'regulation of cell motion' and 'vasculature development' suggests that a complex regulatory network exists between miR-10b and genes pertaining to cell growth, invasion and angiogenesis.

\section{Inhibition of miR-10b decreases invasiveness of U87-2M1} glioma cells. To study miR-10b function in cell invasiveness in vitro, we performed a transwell invasion assay and observed $50 \%$ less invaded U87-2M1 cells after decoy of miR-10b relative to control decoy (Figure 2a). U87-2M1 cells transduced with control decoy vector form big, infiltrative and highly vascularized tumors in vivo with islands of invading cells (Figure $2 b$ ) while inhibition of miR-10b function in U87$2 \mathrm{M} 1$ resulted in a profoundly smaller tumor that is less vascularized with no evidence of localized invasion (Figure 2b).

To understand the molecular mechanism underlying regulation of invasion by miR-10b, we performed western blotting and observed a clear increase in HOXD10 protein level and a reduction in RHOC and PLAUR protein expression (Figure 2c). Our observation agrees with prior reports on miR$10 \mathrm{~b}$ as a negative regulator of HOXD10 in metastatic breast cancer $^{4}$ and $\mathrm{GBM}^{5}$ We questioned if repression of other invasive proteins contributed to diminished U87-2M1 cell invasion after miR-10b inhibition, and found PLAU, CTNNB1, HGF and MMP13 to be significantly downregulated (Figure 2c). Our results point to a broad suppression of invasive proteins after miR-10b inhibition, most likely through indirect mechanisms.

To rule out that the vast decrease in invading U87-2M1 cells was attributed to apoptotic cell death after miR-10b inhibition, we transfected miR-10b mimics into U87-2M1 cells and observed an over twofold increase in invaded cells (Figure 2d). The increased invasiveness mediated by miR$10 \mathrm{~b}$ overexpression is not related to miR-10b's enhancement of cell viability, as miR-10b-overexpressing U87-2M1 cells did not exhibit better viability after 2 days (Figure 2e). By excluding miR-10b's regulation of cell death as a confounding variable, we conclude that $\mathrm{miR}-10 \mathrm{~b}$ likely regulates the invasiveness of U87-2M1 cells.

Inhibition of miR-10b decreases angiogenicity and enhances apoptosis of U87-2M1 glioma cells. We investigated angiogenicity of U87-2M1 tumor after miR-10b inhibition by performing immunohistochemical staining for endothelial cell marker CD31. U87-2M1 cells pretransduced with control decoy vector showed an enrichment of CD31 cells and a migratory tract of CD31-positive cells with numerous CD31-positive vessels (Figure 3a). Remarkably, U87-2M1 cells transduced with miR-10b decoy vector form a much smaller tumor that is only weakly positive for CD31 (Figure 3a). Culture supernatant from miR-10b decoy vectortransduced U87-2M1 is significantly less potent than that from control decoy vector-transduced U87-2M1 in inducing tubule formation from human vascular endothelial cells, further supporting the decreased angiogenic protein production in U87-2M1 cells (Figure 3b). To appreciate the molecular mechanisms underlying U87-2M1's decreased angiogenicity, we performed western blotting and found a pervasive decrease in the expression of angiogenic proteins such as VEGF, IL8 and TGFB2 (Figure 3c). This evidence further supports our finding that U87-2M1 is an invasive and angiogenic subline of U87 glioma cells.

To further examine the group of angiogenic factors picked up by the above DAVID analysis (Table $1 \mathrm{~b}$ ), we used the cBio Cancer Genomics Portal ${ }^{14}$ to analyze mRNA expression of the angiogenic proteins of interest, in the context of all TCGA GBM cases after stratification into the four subtypes. ${ }^{2}$ All 13 angiogenic proteins downregulated by miR-10b inhibition were largely found to be expressed in the mesenchymal subtype of GBM as defined by Verhaak et al. ${ }^{2}$ (Figure 3d). This panel of 13 proteins is, to a lesser extent, expressed in the classical subtype of GBMs and underexpressed or not as differentially regulated in the neural and proneural subtypes of GBMs (Figure 3d). This finding suggests that the mesenchymal subtype of GBMs has a distinct pro-angiogenic signature that may be coregulated by miR-10b.

We verified miR-10b's suppressive effect on apoptosis in U87-2M1 in vitro by performing propidium iodide staining, cell viability assay, caspase activation assays and western blotting for cleaved caspase levels (Supplementary Figures S2A-D). We also demonstrated that direct administration of miR-10b decoy vector in subcutaneous U87-2M1 tumors induces apoptosis in vivo (Supplementary Figure S2E). Critically, mice receiving intracranial implantation of U872M1 cells pre-transduced with miR-10b decoy vector demonstrated a significant survival benefit (Supplementary Figure $\mathrm{S} 2 \mathrm{~F})$.

Pleiotropic miR-10b regulates a broad range of tumor suppressors. To identify mRNA targets of miR-10b in U87$2 \mathrm{M} 1$, we screened through the list of differentially regulated genes to identify potential tumor suppressors that were upregulated after miR-10b inhibition. The longest mRNA transcript variant for every tumor suppressor gene of interest was scrutinized using $R N A 22^{15}$ to identify miR-10b-binding sites. Based on our earlier finding that CTNNB1 is degraded after miR-10b inhibition, we identified CYLD as one negative regulator of the $\mathrm{Wnt} / \beta$-catenin pathway. ${ }^{16} C Y L D$ is also a known negative regulator of $\mathrm{NF} \kappa \mathrm{B}$ signaling, itself a transcriptional activator of $I L 8 .^{17} C Y L D$ transcript contains two predicted miR-10b-binding sites - CYLD 1854 (nucleotide position 1854-1876 residing in the coding sequence) and CYLD 5302 (position 5302-5324 in the 3' UTR) (Supplementary Figure S3). Likewise, two miR-10b sites were predicted for TP53, two in NOTCH1, five in $\mathrm{PTCH1}$, two in PAX6 and two in FOXO3A (Supplementary Figure S3). Although TP53 was not differentially regulated from 
a
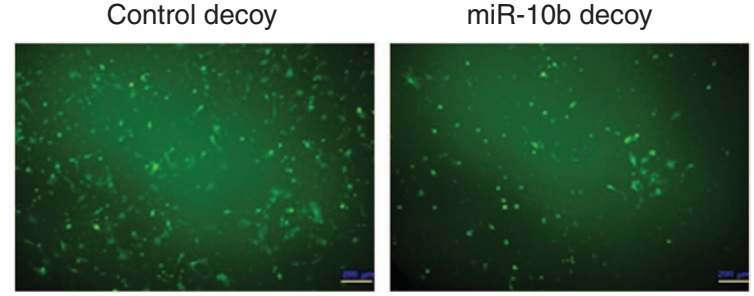

b

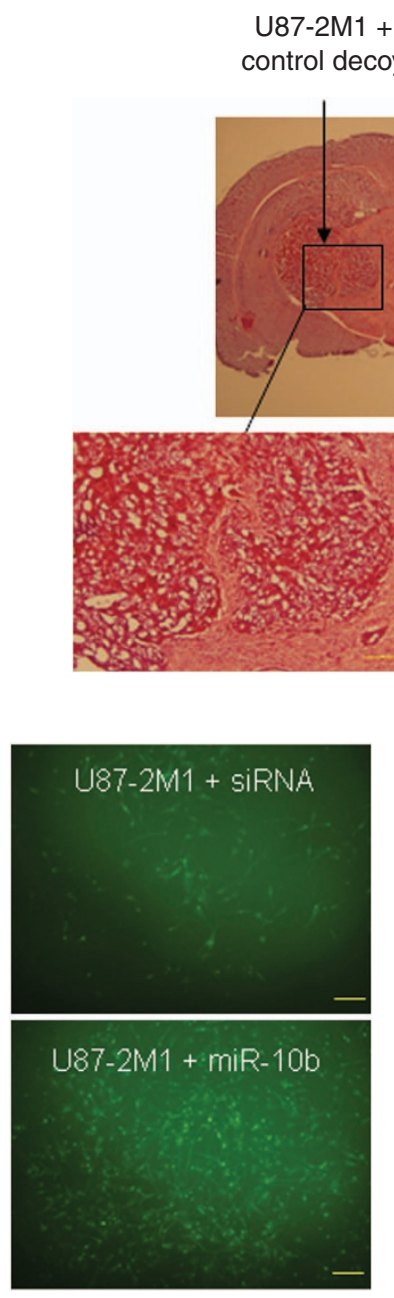

d
miR-10b decoy

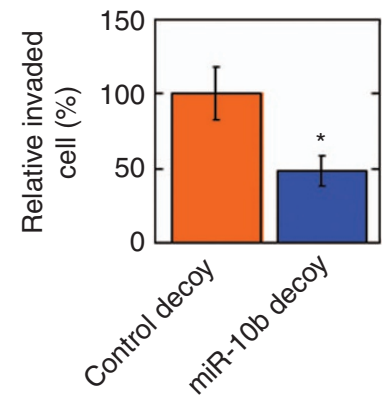

C

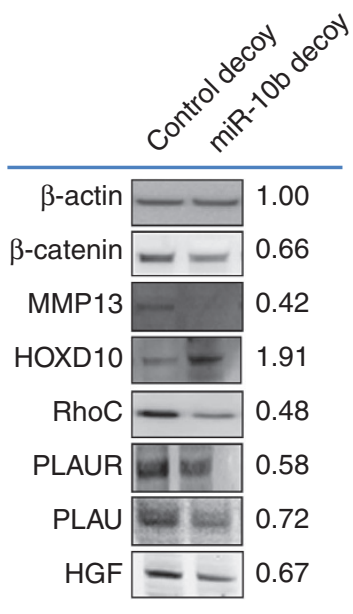

U87-2M1 + $\mathrm{miR}-10 \mathrm{~b}$ decoy 

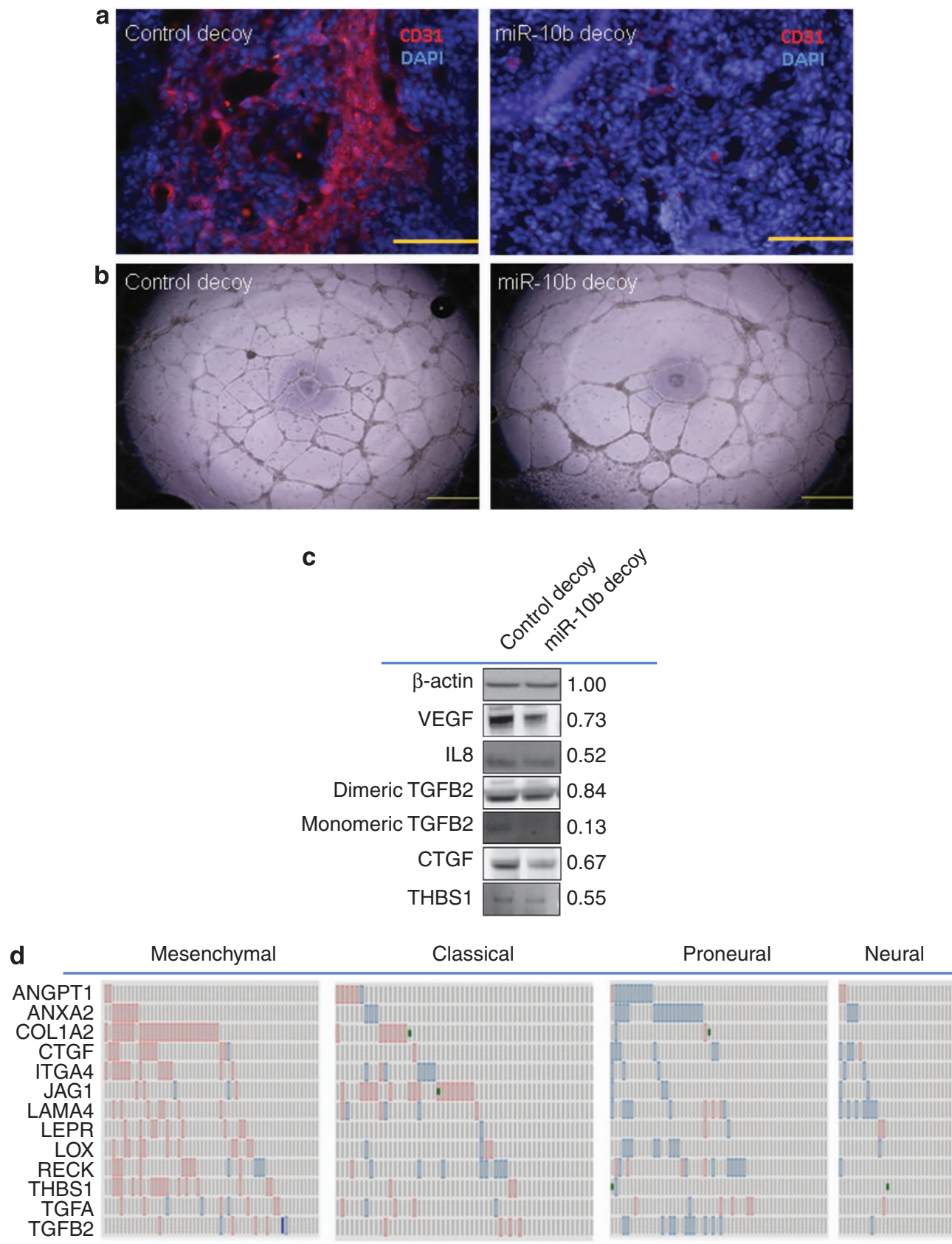

Figure 3 Inhibition of miR-10b suppresses angiogenesis by U87-2M1 glioma cells. (a) In vivo effects. Intracranial tumors produced from miR-10b decoy or control decoy vector-transduced U87-2M1 cells were sectioned and immunostained using antibody against CD31. Bars equal $200 \mu \mathrm{m}$. (b) In vitro effects. Angiogenic potential of U87-2M1 cells were determined by an in vitro endothelial cell tube formation assay. Conditioned medium from U87-2M1 cells transduced with control or miR-10b decoy vector were added to human vascular endothelial cells plated on Matrigel. Bars equal $200 \mu \mathrm{m}$. (c) Downregulation of expression of pro-angiogenic proteins in U87-2M1 cells by miR-10b decoy as demonstrated in western blotting analysis. Numbers indicate relative protein expression in miR-10b decoy-treated U87-2M1 cells compared with control decoytransduced U87-2M1 cells after normalization to beta-actin levels. Blots shown are representative of biological triplicates. (d) A panel of 13 angiogenic genes downregulated by miR-10b silencing is frequently overexpressed in the Mesenchymal subtype of GBM. Most of the genes in this panel are less frequently overexpressed in the Classical subtype of GBM, and frequently underexpressed in the 'Neural' and 'Proneural' subtypes of GBM. Oncoprints involving the panel of 13 angiogenic genes in the Mesenchymal, Classical, Neural and Proneural subtypes of GBM were derived from the cBio Cancer Genomics Portal. ${ }^{14}$ In each oncoprint, individual genes are represented as rows and individual patients or cases are represented as columns. The stratification of the patients and cases from TCGA GBM was done according to the molecular classification defined by Verhaak et al. ${ }^{2}$ Red-outlined boxes indicate mRNA overexpression and red-filled boxes indicate amplification. Blue-outlined boxes indicate mRNA underexpression and blue-filled boxes indicate homozygous deletions. Green boxes indicate presence of gene mutation

miR-10b-binding sites in the region important for pairing with miR-10b's seed sequence and observed amelioration of miR10b-mediated luciferase repression in seven mutated binding sites (Figure 4a). Endogenous CYLD, TP53, FOXO3A, PAX6, NOTCH1 and PTCH1 protein levels were assayed in U87-2M1 cells after silencing of miR-10b. In agreement, 


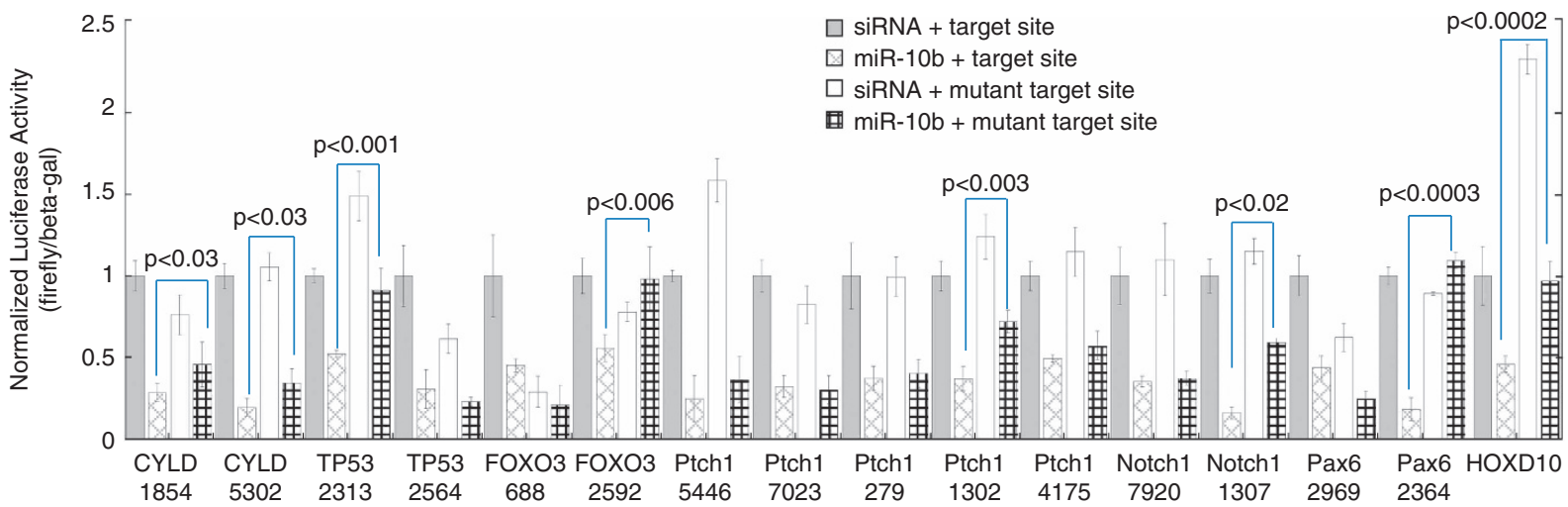

b

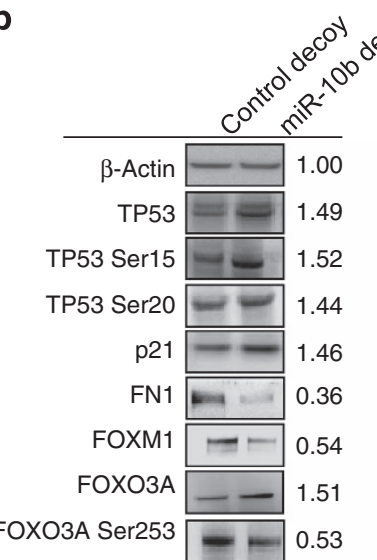

C

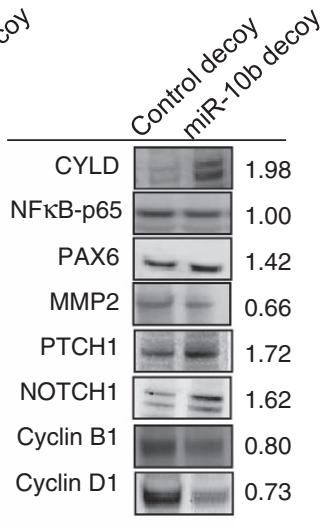

d

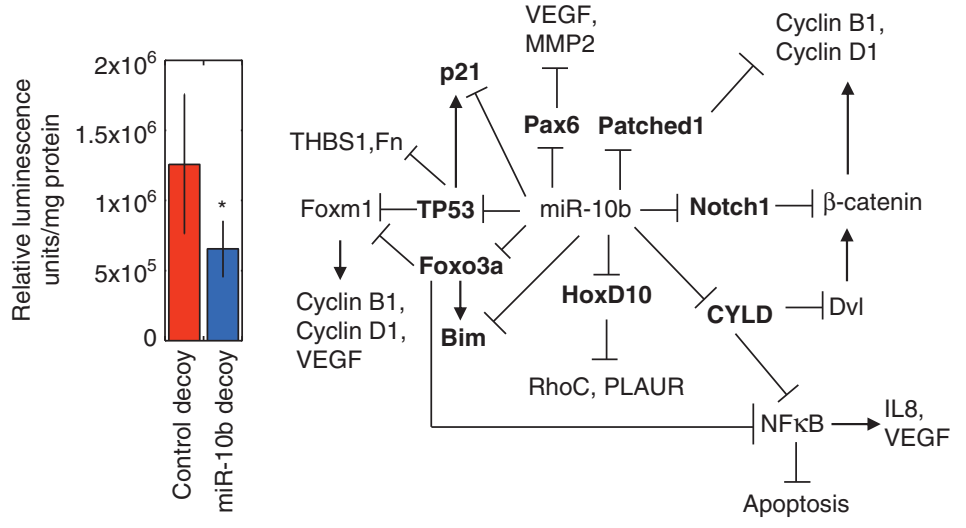

Figure 4 Identification of TP53, FOXO3, CYLD, NOTCH1, PTCH1 and PAX6 as targets of miR-10b. (a) Response of luciferase reporters harboring either the predicted miRNA target site or its corresponding mutant form, after codelivery of miR-10b or control siRNA into MCF7 cells. Error bars represent S.D. from three to four independent transfections. (b) Silencing of miR-10b upregulates target proteins TP53, FOXO3, CYLD, PTCH1, NOTCH1 and PAX6. Regulation of proteins downstream of TP53, FOXO3, CYLD, PTCH1, NOTCH1 and PAX6 are shown. Numbers indicate relative protein expression in miR-10b decoy-treated U87-2M1 cells compared with control decoytransduced U87-2M1 cells after normalization to beta-actin levels. Blots shown are representative of biological triplicates. (c) NF $\kappa$ B transcriptional activity is significantly lower in miR-10b-silenced U87-2M1 cells. Error bars represent S.E. from three independent experiments; ${ }^{*} P<0.05$. (d) A mechanistic model summarizing the pleiotropic actions of miR-10b

inhibition of miR-10b enhanced the endogenous protein expression of these six predicted targets in U87-2M1 cells (Figure 4b). These data indicate that miR-10b directly regulates endogenous CYLD, TP53, FOXO3A, PAX6, NOTCH1 and PTCH1.

CYLD has a dampening effect of $\mathrm{NF} \kappa \mathrm{B}$ pathway activation, which in itself exerts angiogenic properties and protects against apoptosis among its many roles. ${ }^{18}$ CYLD's deubiquitination of $\mathrm{NF}_{\kappa} \mathrm{B}$ inhibits $\mathrm{NF}_{\kappa} \mathrm{B}$ activity without decreasing $\mathrm{NF} \kappa \mathrm{B}$ protein expression. ${ }^{19,20}$ Concurring, we observed a decrease in $\mathrm{NF} \kappa \mathrm{B}$ transcriptional activity (Figure $4 \mathrm{c}$ ) in the absence of a downregulation of NF $\kappa \mathrm{B}-\mathrm{p} 65$ expression (Figure 4b) and consequently, decreased expression of IL8 and VEGF (Figure 3c). Increased CYLD expression may likewise diminish Wnt pathway activation and $\beta$-catenin accumulation (Figure 2c) via K63-linked deubiquitination of Dvl. ${ }^{16}$ Decreased CTNNB1 expression could also be a consequence of enhanced NOTCH1 expression (Figure 4b), which has a demonstrated tumor suppressive function in mouse skin. ${ }^{21}$ In addition, Sonic hedgehog (Shh) and Wnt pathways collaborate to regulate cell cycle progression via the production of early and late cyclins in neural progenitor cells. ${ }^{22}$ Therefore, increased expression of PTCH1 (Figure 4b) could exert a suppressive effect on Shh pathway and act synergistically with loss of CTNNB1 expression to halt cell cycle progression. CTNNB1's oncogenic role may be augmented by the expression of FOXM1 in glioblastoma. ${ }^{23}$ FOXM1 is overexpressed in glioblastomas, ${ }^{24}$ transcribes $\mathrm{VEGF}^{13}$ and critically mediates $\beta$-catenin's nuclear translocation in the absence of conventional Wnt pathway activation. ${ }^{23}$ Others have shown that $\mathrm{TP}^{2} 3^{24}$ and $\mathrm{FOXO}^{25}$ negatively regulate FOXM1. In concurrence, FOXM1 protein expression is downregulated together with upregulation of TP53 and FOXO3 (Figure 4b) after inhibition of miR-10b. Upregulation of $\mathrm{FOXO} 3$ activates pro-apoptotic Bim, which is itself a target of miR-10b. ${ }^{3}$ In addition, upregulation of FOXO3 after miR$10 \mathrm{~b}$ silencing provides an alternative mechanistic explanation for diminished NF $\kappa$ B activity. ${ }^{26}$ TP53's activation may activate p21 expression ${ }^{27}$ and inhibit expression of fibronectin ${ }^{28}$ and thrombospondin $1,{ }^{29}$ which were observed after miR-10b silencing (Figure 4b). Furthermore, phosphorylation of TP53 at Ser15 and Ser20 reduces Mdm2's ability to negate TP53 
activity (Figure 4b). Finally, PAX6 upregulation (Figure 4b) may suppress angiogenicity and invasiveness owing to PAX6's repression of VEGFA ${ }^{30}$ and MMP2. ${ }^{31}$

Our findings point to a complex regulatory framework centered on miR-10b in glioma cells (Figure 4d). miRNA-10b coordinately downregulates many tumor suppressors, resulting in the suppression of cell cycle inhibitors while promoting expression of invasive and angiogenic molecules.

miRNA-10b's target genes HOXD10, NOTCH1, TP53 and $P A X 6$ regulate invasiveness of U87-2M1 glioma cells. To assess the contribution of the identified tumor suppressors on U87-2M1's invasiveness, we transfected miR-10b decoy vector-transduced U87-2M1 cells with pools of siRNA against each of the identified tumor suppressors regulated by miR-10b. Efficient knockdown of CYLD, TP53, FOXO3A, PAX6, NOTCH1, PTCH1 and HOXD10 is demonstrated using western blotting (Figure $5 \mathrm{a}$ ). In transwell invasion assays, knockdown of TP53, PAX6, NOTCH1 and HOXD10 significantly ameliorated suppression of invasion mediated by miR-10b inhibition (Figure $5 b$ ). This is not unexpected given that a single miRNA is known to modulate several targets in its regulation of cellular processes. In contrast, knockdown of CYLD, FOXO3A and PTCH1 did not significantly alter the invasion capacity of U87-2M1 (Figure 5b).

Perturbation of direct and indirect targets of miR-10b correlates with poorer patient survival. To determine the clinical importance of various miR-10b-regulated tumor suppressors, we assessed the relevant patient survival rates by interrogating the REMBRANDT database. ${ }^{32}$ At a foldchange threshold of 1.5, reduced expression of PAX6, CYLD, FOXO3, PTCH1 and NOTCH1 were associated with significantly lower patient survival (Figure 6a). A lack of association between diminished p21, TP53, BIM, HOXD10 and patient survival (Figure 6a) may be attributed to the prevalence of mutational inactivation in tumor suppressors that do not necessarily compromise mRNA expression. ${ }^{1}$ More conclusively, all oncogenic proteins indirectly regulated by miR-10b are significantly associated with poorer patient survival at a fold-change threshold of 1.5 (Figure $6 \mathrm{~b}$ ).

\section{Discussion}

In the present report, we derived an invasive subline of U87 cells, U87-2M1. Enhanced malignancy of U87-2M1 cells as opposed to parental U87 cells could be in part owing to increased expression of miR-10b. A baculoviral vector encoding decoy miR-10b-binding sites was found to sufficiently silence miR-10b activity, resulting in the detection of broad changes in U87-2M1 cells from cell growth, invasion to angiogenicity.

miRNA-10b's pleiotropic action in this study stood in direct contrast to earlier reports on miR-10b's exclusive regulation of either glioma cell growth ${ }^{3}$ or glioma invasion. ${ }^{5}$ Although miR10b's impact on cell cycle and apoptosis may appear to confound our findings on miR-10b's regulation of glioma invasion, miR-10b gain-of-function studies showed that miR$10 \mathrm{~b}$ is able to positively regulate cell invasion. In addition, invasive proteins such as MMP13, MMP2, CTNNB1 and HGF
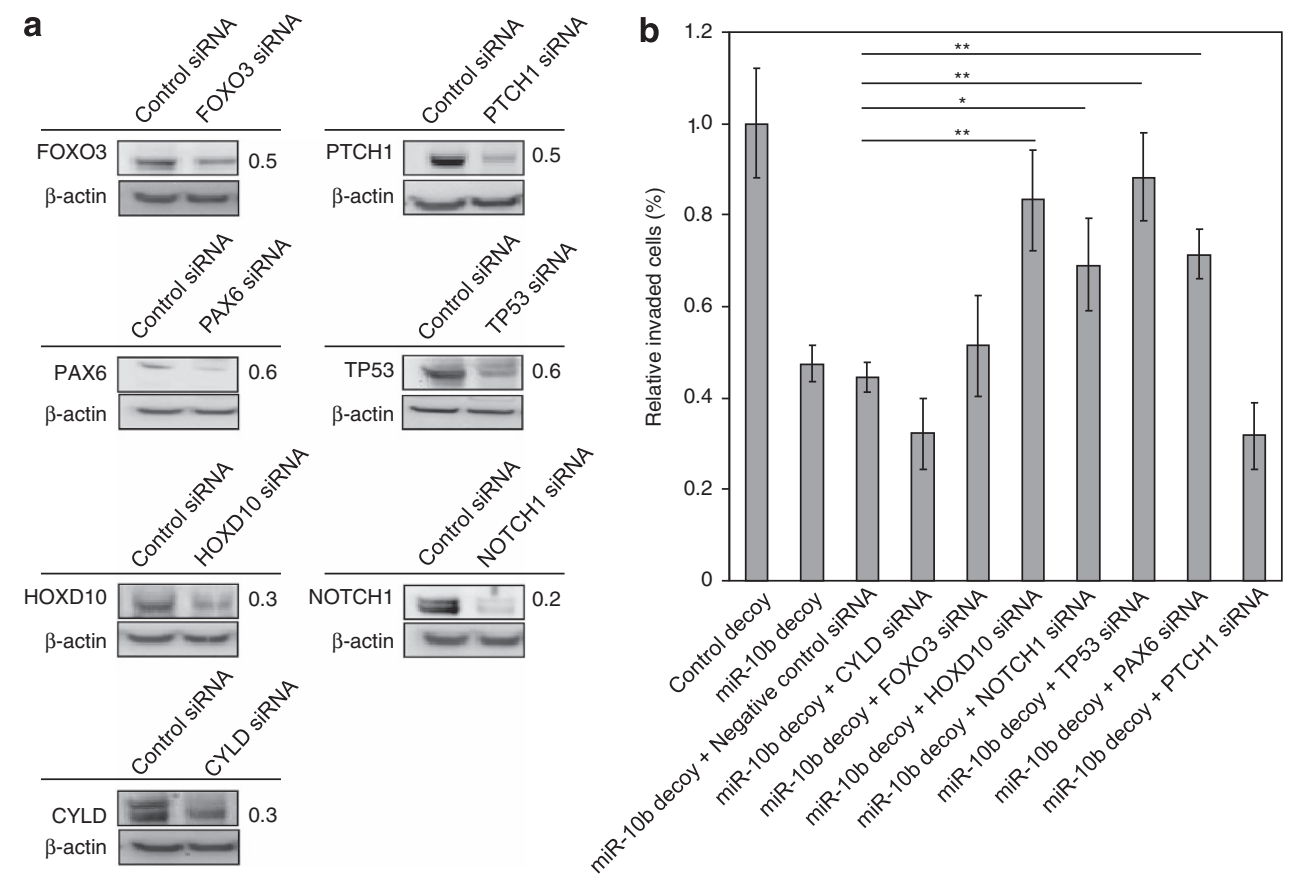

Figure 5 HOXD10, NOTCH1, TP53 and PAX6 regulate invasiveness of U87-2M1 glioma cells. (a) Western blotting shows that the gene-specific siRNA pools were effective in knocking down protein levels of TP53, FOXO3, CYLD, PTCH1, NOTCH1, HOXD10 and PAX6. Numbers indicate relative protein expression in gene-specific siRNA-treated U87-2M1 cells compared with negative control siRNA-treated U87-2M1 cells after normalization to beta-actin levels. Blots shown are representative of biological triplicates. (b) siRNA-mediated knockdown of miR-10b's targets HOXD10, NOTCH1, TP53 and PAX6, but not CYLD, PTCH1 or FOXO3, ameliorate diminished U87-2M1 invasion in vitro after miR-10b inhibition. Error bars represent S.E. from three independent experiments; ${ }^{\star} P<0.05$; ${ }^{\star \star} P<0.01$ 
a

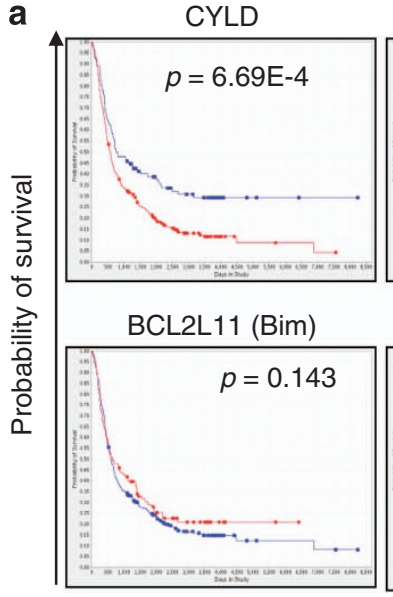

FOXO3

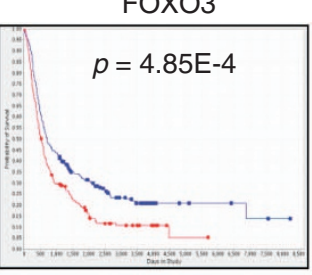

CDKN1A (p21)

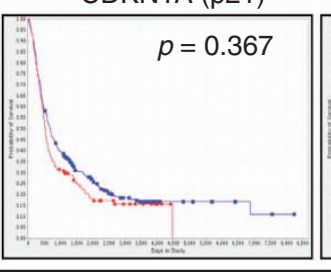

NOTCH1

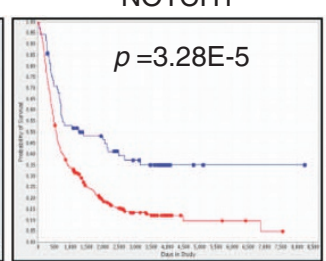

TP53

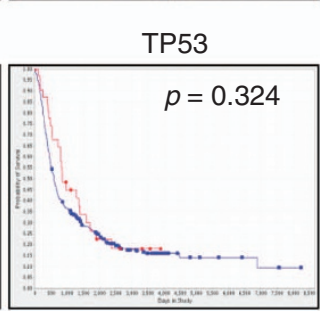

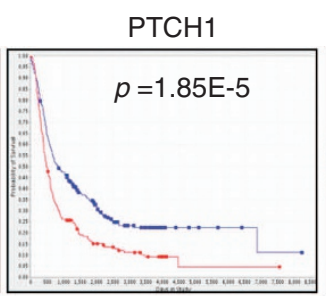

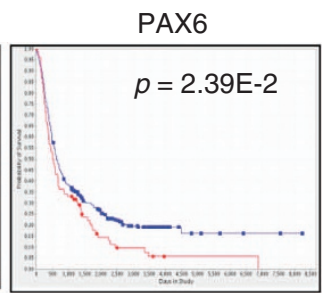

HOXD10

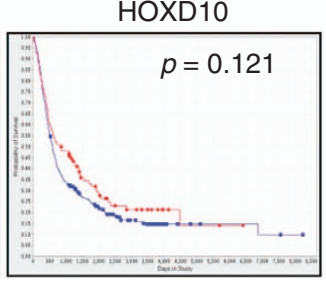

Days in study

b

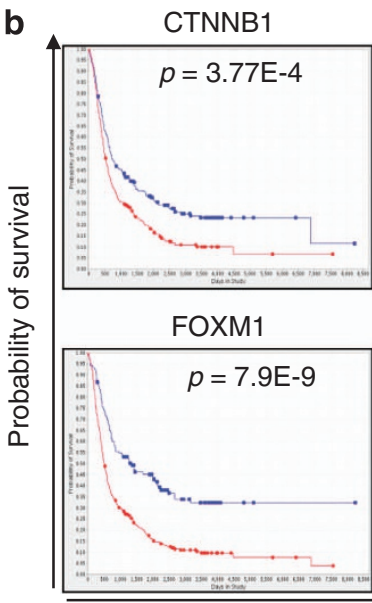

NF $\kappa$ B1

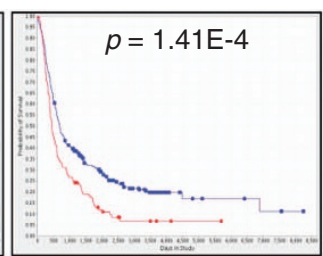

$\mathrm{RHOC}$

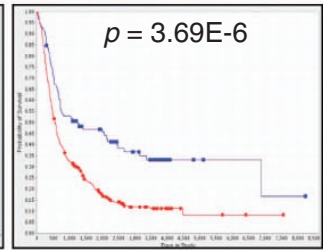

IL8

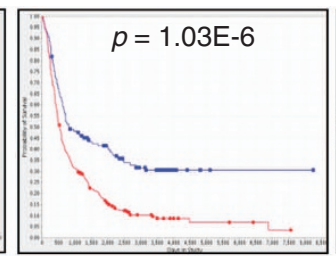

VEGFA

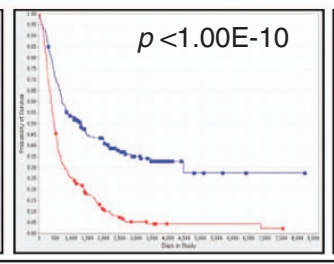

Days in study

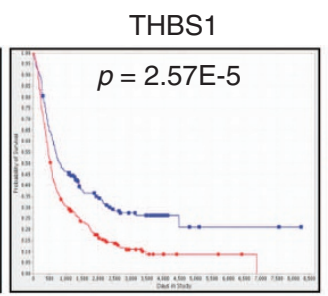

MMP2
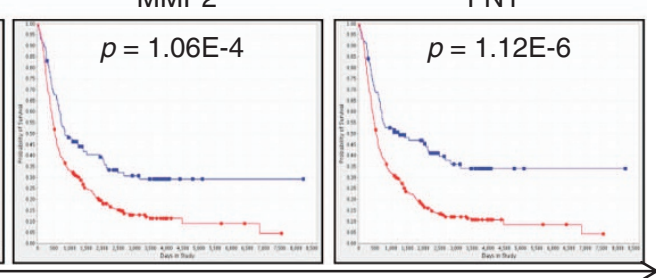

PLAUR

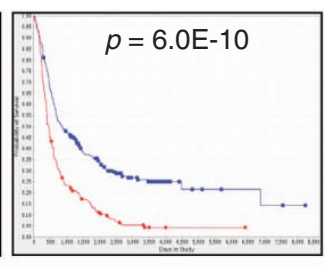

FN1 $\longrightarrow$

Figure 6 Perturbed expression of direct and indirect targets of miR-10b significantly correlates with poor patient survival. Patient survival rates were assessed by interrogating the REMBRANDT. (a) Most of the direct targets of miR-10b are associated with poor patient survival. Red line denotes survival curve of patients with $>1.5$-fold underexpression of the gene of interest. Blue line denotes survival curve of patients with $<1.5$-fold underexpression of the gene of interest. (b) Indirect oncogenic targets of miR-10b significantly correlate with poor patient survival. Red line denotes survival curve of patients with $>1.5$-fold overexpression of the gene of interest. Blue line denotes survival curve of patients with $<1.5$-fold overexpression of the gene of interest

were downregulated after miR-10b silencing, supporting miR10b's regulation of glioma invasion. Several laboratories have undertaken efforts using unsupervised hierarchical clustering to delineate gene expression signatures that are preferentially activated in different subtypes of $\mathrm{GBM},{ }^{2,33}$ suggesting that the biological behavior and malignancy of each GBM subtype is likely different. We noted that the lack of correlation between miR-10b and glioma invasion was made in the context of all glioblastoma cases under the TCGA study, ${ }^{3}$ where only $<30 \%$ of all glioblastoma cases were categorized as having a mesenchymal signature. ${ }^{2,34}$ Although miR-10b may universally regulate glioma cell growth and death regardless of the GBM subtype being studied, it seems plausible that additional roles for miR-10b may arise depending on the repertoire of mRNA expression. In our study, we have shown that HOXD10, PAX6, TP53 and NOTCH1 may coordinately regulate invasion via suppression of proteins such as $\mathrm{RHOC}$, PLAUR, MMP2 and CTNNB1.
Other than governing invasiveness of U87-2M1 glioma cells, we have also shown that miR-10b displays the same propensity toward regulation of U87-2M1 cell death and growth. This challenges the notion that miR-10b exclusively performs a singular role of either promoting cell invasion ${ }^{4}$ or cell growth. ${ }^{3}$ Of particular interest, Ma et al. ${ }^{4}$ observed a significant increase in the weight of SUM149 mammary tumors stably expressing miR-10b as opposed to control tumors from the ninth week of SUM149 xenograft development. Although the authors suggested that the invasiveness of the cells provided better access to vasculature that aid tumor development, we reckon the development of tumors in vivo provided microenvironmental signals, for example hypoxia, which may modulate the repertoire of mRNA expression and allow miR$10 \mathrm{~b}$ to simultaneously promote cancer cell growth, angiogenesis and invasion while suppressing apoptosis.

We have demonstrated a previously unappreciated role for $\mathrm{miR}-10 \mathrm{~b}$ in regulating glioma angiogenesis. This is not 
improbable as many proteins are pleiotropic suppressors of tumorigenesis. Furthermore, Ma et al. ${ }^{4}$ reported the finding of highly proliferative and angiogenic SUM149 mammary cancer cells at the invasive fronts of an in vivo SUM149 mammary tumor stably expressing miR-10b, suggesting that it is possible for miR-10b to act in a pleiotropic manner that was previously unknown. Microarray analyses revealed a panel of 13 angiogenic molecules that was downregulated after miR$10 \mathrm{~b}$ silencing. Out of these 13 proteins, 11 of them have been implicated in glioma malignancy and angiogenesis. ANGPT1, ${ }^{35}$ COL1A2, JAG1, ITGA4, LAMA4, THBS1 and TGFB2, ${ }^{36}$ CTGF, ANXA2 and LOX,${ }^{37}$ and TGFA ${ }^{38}$ have been shown to underlie the angiogenic, invasive or mesenchymal gene expression of glioma cells while LEPR is a key receptor for leptin-mediated angiogenesis in acute myeloid leukemia. ${ }^{39}$ RECK is a well-characterized MMP-dependent anti-angiogenic molecule whose expression in U87-2M1 cells seems paradoxical. Interestingly, RECK has been shown to possess an MMP-independent function. Osteoarthritic chondrocytes proliferating in response to TGF- $\beta$ required the presence of RECK and silencing of RECK-reduced proliferation of TGF- $\beta$ stimulated chondrocytes. ${ }^{38}$ This finding suggests that RECK and TGF- $\beta$ coordinately and positively regulates cell proliferation in an inflammatory setting. As the mesenchymal subtype of GBM is characterized by a high expression of genes from the NF- $\kappa$ B pathway, RECK likely has a pro-proliferative, rather than anti-angiogenic, role in U87-2M1 cells. Moreover, RECK overexpression is associated with poor patient survival rate in the REMBRANDT dataset $(P=0.002)$, suggesting that RECK is a glioma oncogene (Supplementary Figure S4). Similarly, THBS1 has been widely regarded as a tumor suppressor via its inhibition of angiogenesis. However, patient survival data from REMBRANDT database reveals a highly significant link between THBS1 overexpression and poor survival rates (Figure 6b). ${ }^{32}$ We speculate that THBS1 acts as an oncogene via activation of latent TGF- $\beta$ in the extracellular environment. ${ }^{40}$ Downregulation of pro-angiogenic Notch ligand, JAG1, enables NOTCH1 receptor to act as a tumor suppressor via its sequestration of CTNNB1, hence avoiding conflict with its potential angiogenic contribution via JAG1 activation. Our results collectively indicate a reduced angiogenicity of U87-2M1 glioma cells after miR-10b silencing.

Our findings further confirmed the utility of miR-10b silencing for glioma therapy. Although miR-10b has been established to regulate cell growth and apoptosis across all gliomas, miR-10b's role should be expanded to include regulation of angiogenesis and invasion, at least, in the mesenchymal subtype of gliomas. Whether miR-10b possesses such a pleiotropic role in other subtypes of glioma requires development of in vitro models that accurately mimic other GBM subtypes.

\section{Materials and Methods \\ Cell culture. U87, U251, U138, SW1088 and T98G were obtained from American Type Culture Collection (Manassas, VA, USA). NHA and human umbilical venous endothelial cells (HUVEC) were from Lonza (Basel, Switzerland). U87-2M1 was derived from U87 cells via an ELM assay as described ${ }^{8}$ and propagated in vitro under hypoxic conditions ( $94 \%$ nitrogen, $5 \%$ carbon dioxide and $1 \%$ oxygen).}

Baculovirus preparation. Oligos harboring 13 repeats of a miR-10bbinding site or control-binding site were synthesized, phosphorylated, annealed and ligated downstream of a CMV-eGFP cassette. Recombinant baculoviral vectors were generated using the Bac-to-Bac baculovirus expression system (Life Technologies, Carlsbad, CA, USA). Transduction of glioma cells were performed at a multiplicity of infection of 200 .

Glioma models. To analyze the difference between U87 and U87-2M1 orthotopic growth trends, $2 \times 10^{5}$ U87-2M1 or U87 cells were inoculated intracranially in Balb c/nude mice. After 3 weeks of tumor growth, brain tissues were sectioned and H\&E staining was performed. To elucidate the effects of miR$10 \mathrm{~b}$ silencing on intracranial growth of U87-2M1 cells, $2 \times 10^{5}$ U87-2M1 cells transduced with either control decoy vector or miR-10b decoy vector were injected into the left and right striatum, respectively, in nude mice. Brain tissues were collected after 3 weeks of tumor growth. H\&E staining or CD31 (Abcam, Cambridge, UK) immunohistochemical staining was performed subsequently. Subcutaneous U87-2M1 tumors were established by injecting $2 \times 10^{6}$ cells into the subcutaneous space. Plaque-forming units of control $\left(10^{8}\right)$ or miR-10b decoy baculoviral vectors were injected into the subcutaneous tumors. For survival analyses, U87-2M1 cells in vitro were either untransduced or transduced with control decoy vector or miR-10b decoy vector. Each mouse in the three groups of 10 mice was inoculated intracranially with $2 \times 10^{5}$ U87-2M1 and monitored every day.

Western blot and quantification. The antibodies used were antiFibronectin, anti-Twist, anti-Snai1, anti-Snai2, anti-FOXM1, anti-PLAUR, anti- $\beta$ catenin, anti-IL8, anti-TP53, anti-MMP2, anti-Ptch1, anti-Cyclin B1 and anti-Cyclin D1 from Santa Cruz Biotechnology (Santa Cruz, CA, USA); anti-E-cadherin, anti-N-cadherin and anti-VEGF from BD Pharmingen (San Diego, CA, USA); anti$\beta$-Actin, anti-Vimentin, anti-CTGF, anti-THBS1, anti-HOXD10, anti-Notch1 and anti-MMP13 from Abcam; anti-Stat3, anti-Cleaved Caspase-3, anti-Cleaved Caspase-7, anti-Bim, anti-TP53-S15, anti-TP53-S20, anti-p21, anti-FOXO3, antiFOXO3-S253, anti-pan-Akt, anti-CYLD and anti-NF $\kappa B$ from Cell Signaling (Danvers, MA, USA); anti-HGF, anti-PLAU, anti-TGFB2 and anti-PAX6 from Novus Biologicals (Littleton, CO, USA) and anti-RhoC from Abnova (Taipei, Taiwan). Western blot bands were subjected to densitometry analyses using Image J (National Institutes of Health, Bethesda, MD, USA). Mean relative intensities of the relevant protein bands were quantified from triplicates and normalized to beta-actin levels (Supplementary Figures S5-S7). Western blots from full-length gels are displayed in (Supplementary Figures S8-S12). Representative blots for the relevant proteins are shown in Figures 1-5 and Supplementary Figure S2.

miRNA quantification. Total RNA was extracted using TRIzol (Life Technologies) and miRNA quantification performed using the Taqman miRNA reverse transcription and Taqman miRNA assays according to manufacturer's instructions (Life Technologies).

Microarray and bioinformatics data analysis. Gene expression profiling was performed on the Genechip Human Genome U133 Plus 2.0 platform (Affymetrix) in accordance with the manufacturer's protocol. Statistical analyses and data normalization were carried out using the Genespring GX software (Agilent Technologies, Santa Clara, CA, USA). For comparing difference in gene expression of U87 and U87-2M1 glioma cells, genes with fold change $>2$ were deemed to be differentially expressed. Genes with fold change $>1.5$-fold were deemed to be differentially regulated by miR-10b silencing in U87-2M1 cells. The data discussed in this publication have been deposited in NCBl's Gene Expression Omnibus and are accessible through GEO SuperSeries accession number GSE 35208. Gene ontology categories were analyzed using DAVID version $6.7 .^{10}$ Angiogenic genes were analyzed in the cBio Cancer Genomics Portal (accessed December 2011) ${ }^{14}$ using an expression z-score threshold of 1.5 and a selection of patient/case set of either the classical, mesenchymal, neural or proneural subtypes of GBM.

For the generation of heatmaps, genes used in the classification of the four GBM gene expression subtypes were downloaded from Verhaak et al.'s publication, ${ }^{2}$ and microarray probes that correspond to these genes and are common to both the Affymetrix HG U133A platform (used by TCGA Research Network) and HG U133 Plus 2.0 platform (used in our study) were identified. Normalized probe intensity values were extracted and analysis was performed using Genespring GX (Agilent Technologies). 
Invasion assay. U87-2M1 glioma cells were either transduced with control or miR-10b decoy vectors (for miR-10b inhibition studies), or transfected with $50 \mathrm{nmol} / / \mathrm{l}$ negative control siRNA (Qiagen, Hilden, Germany) or miR-10b mimic (Qiagen) (for miR-10b overexpression studies) for $24 \mathrm{~h}$. For miR-10b overexpression studies, cells were labeled with Calcein AM dye (Life Technologies) before seeding into transwell chambers for subsequent visualization. Rescue experiments were performed after transduction of cells with miR-10b decoy vector and transfection with gene-specific siRNA pools at $20 \mathrm{nM}$. After cells were subjected to the required treatment, 50000 cells in serum-free media, Opti-Mem (Life Technologies), were seeded in each transwell chamber, $8.0 \mu \mathrm{m}$ Fluorobok Insert (BD Falcon, San Jose, CA, USA) coated with the basement membrane matrix, Geltrex (Life Technologies), and cells were allowed to invade for $24 \mathrm{~h}$ toward serum-containing media. Invading cells in treatment and control groups were imaged using fluorescent microscopy and quantified. Number of invading cells in the control group was taken to be $100 \%$. Invading cells in the treatment groups were compared with the respective control group taken at $100 \%$. Experiments were carried out in sextuplicates.

Endothelial tubule formation assay. HUVEC $\left(2.5 \times 10^{4}\right)$ were grown on Matrigel-coated 96-well plates in the presence of $100 \mu \mathrm{l}$ of conditioned medium from $3 \times 10^{5}$ U87-2M1 glioma cells. HUVEC were allowed to incubate for $24 \mathrm{~h}$ at $37^{\circ} \mathrm{C}$ before imaging.

Cell cycle, cell proliferation/viability and caspase assays. For cell cycle analysis, attached and floating U87-2M1 cells were collected 3 days following transduction for propidium iodide staining and analyzed on the LSRII flow cytometry analyzer (BD Biosciences, San Jose, CA, USA).

For cell proliferation or viability assay, U87-2M1 cells were transduced and assayed using CellTiter96 AQueous One Solution Cell Proliferation Assay (Promega, Madison, WI, USA). Caspase- 3 and caspase-7 activities were measured $48 \mathrm{~h}$ post transduction using the Caspase-Glo 3/7 Assay (Promega).

miRNA target prediction. Longest transcripts for TP53 (variant 1), Notch1, Ptch1 (variant 1b), Pax6 (variant 1), FOXO3 (variant 1) and CYLD (variant 1) were retrieved from NCBl's 'Nucleotide' database and screened using rna22 ${ }^{14}$ for miR-10b-binding sites. We did not allow for any unpaired bases in a seed of six nucleotides permitted a maximum folding energy of $-15 \mathrm{Kcal} / \mathrm{mol}$.

Predicted miRNA target sites, including seven nucleotides flanking each site, were synthesized as oligomers, phosphorylated and cloned into pMIR-REPORT Luciferase reporter vector (Life Technologies). MCF7 cells were cotransfected with pMIR-REPORT Luciferase reporter vector, beta-galactosidase reporter vector and with either Allstars negative control siRNA (Qiagen) or miR-10b mimic (Qiagen). Luciferase activity and beta-galactosidase activity were quantified using the Luciferase Assay System (Promega) and Beta-galactosidase Enzyme Assay System (Promega). Luciferase activity was normalized to beta-galactosidase activity.

NF $\boldsymbol{k}$ B promoter activity assay. NF- $\kappa \mathrm{B}$ promoter activity was assayed by transfecting U87-2M1 cells with the pGL4.32[luc2P/NF- $\kappa$ B-RE/Hygro] vector (Promega) and transducing cells with either control decoy vector or miR-10b decoy vector. Luciferase activity was normalized to cellular protein levels.

Statistical analysis. All data are represented as mean \pm S.E.M. Differences between samples were analyzed using Student's $t$-test. The statistical analysis of survival data was performed using the log-rank test followed by Holm-Sidak method for pairwise multiple comparison tests. A $P$-value $<0.05$ was considered to be statistically significant.

\section{Conflict of Interest}

The authors declare no conflict of interest.

Acknowledgements. We are grateful to the members of Wang Shu's group, especially Yukti Choudhury, for their help and suggestions. This research was supported by a grant from Singapore Ministry of Education (MOE2011-T2-1-056) and Institute of Bioengineering and Nanotechnology, Biomedical Research
Council, Agency for Science, Technology and Research (A*STAR) in Singapore (IBN/04-R12002).

1. Network TCGAR. Comprehensive genomic characterization defines human glioblastoma genes and core pathways. Nature 2008; 455: 1061-1068.

2. Verhaak RG, Hoadley KA, Purdom E, Wang V, Qi Y, Wilkerson MD et al. Integrated genomic analysis identifies clinically relevant subtypes of glioblastoma characterized by abnormalities in PDGFRA, IDH1, EGFR, and NF1. Cancer Cell 2010; 17: 98-110.

3. Gabriely G, Yi M, Narayan RS, Niers JM, Wurdinger T, Imitola J et al. Human glioma growth is controlled by microRNA-10b. Cancer Res 2011; 71: 3563-3572.

4. Ma L, Teruya-Feldstein J, Weinberg RA. Tumour invasion and metastasis initiated by microRNA-10b in breast cancer. Nature 2007; 449: 682-688.

5. Sun L, Yan W, Wang Y, Sun G, Luo H, Zhang J et al. MicroRNA-10b induces glioma cell invasion by modulating MMP-14 and uPAR expression via HOXD10. Brain Res 2011; 1389: 9-18.

6. Chai G, Liu N, Ma J, Li H, Oblinger JL, Prahalad AK et al. MicroRNA-10b regulates tumorigenesis in neurofibromatosis type 1. Cancer Sci 2010; 101: 1997-2004.

7. Singh SK, Hawkins C, Clarke ID, Squire JA, Bayani J, Hide T et al. Identification of human brain tumour initiating cells. Nature 2004; 432: 396-401.

8. Xie Q, Thompson R, Hardy K, DeCamp L, Berghuis B, Sigler R et al. A highly invasive human glioblastoma pre-clinical model for testing therapeutics. J Transl Med 2008; 6: 77.

9. Wang CY, Li F, Yang Y, Guo HY, Wu CX, Wang S. Recombinant baculovirus containing the diphtheria toxin A gene for malignant glioma therapy. Cancer Res 2006; 66: 5798-5806.

10. Huang da W, Sherman BT, Lempicki RA. Systematic and integrative analysis of large gene lists using DAVID bioinformatics resources. Nat Protoc 2009; 4: 44-57.

11. Carro MS, Lim WK, Alvarez MJ, Bollo RJ, Zhao X, Snyder EY et al. The transcriptional network for mesenchymal transformation of brain tumours. Nature 2010; 463: 318-325.

12. Inoue A, Takahashi H, Harada H, Kohno S, Ohue S, Kobayashi K et al. Cancer stem-like cells of glioblastoma characteristically express MMP-13 and display highly invasive activity. Int J Oncol 2010; 37: 1121-1131.

13. Zhang Y, Zhang N, Dai B, Liu M, Sawaya R, Xie K et al. FoxM1B transcriptionally regulates vascular endothelial growth factor expression and promotes the angiogenesis and growth of glioma cells. Cancer Res 2008; 68: 8733-8742.

14. The cBio Cancer Genomics Portal (December 2011). Available from: http://www. cbioportal.org/.

15. Miranda KC, Huynh T, Tay Y, Ang YS, Tam WL, Thomson AM et al. A pattern-based method for the identification of MicroRNA binding sites and their corresponding heteroduplexes. Cell 2006; 126: 1203-1217.

16. Tauriello DV, Haegebarth A, Kuper I, Edelmann MJ, Henraat M, Canninga-van Dijk MR et al. Loss of the tumor suppressor CYLD enhances Wnt/beta-catenin signaling through K63-linked ubiquitination of Dvl. Mol Cell 2010; 37: 607-619.

17. Kunsch C, Lang RK, Rosen CA, Shannon MF. Synergistic transcriptional activation of the IL-8 gene by NF-kappa B p65 (RelA) and NF-IL-6. J Immunol 1994; 153: 153-164.

18. Yoshida S, Ono M, Shono T, Izumi H, Ishibashi T, Suzuki $\mathrm{H}$ et al. Involvement of interleukin-8, vascular endothelial growth factor, and basic fibroblast growth factor in tumor necrosis factor alpha-dependent angiogenesis. Mol Cell Biol 1997; 17: 4015-4023.

19. Kovalenko A, Chable-Bessia C, Cantarella G, Israel A, Wallach D, Courtois G. The tumour suppressor CYLD negatively regulates NF-kappaB signalling by deubiquitination. Nature 2003; 424: 801-805.

20. Trompouki E, Hatzivassiliou E, Tsichritzis T, Farmer H, Ashworth A, Mosialos G. CYLD is a deubiquitinating enzyme that negatively regulates NF-kappaB activation by TNFR family members. Nature 2003; 424: 793-796.

21. Nicolas M, Wolfer A, Raj K, Kummer JA, Mill P, van Noort M et al. Notch1 functions as a tumor suppressor in mouse skin. Nat Genet 2003; 33: 416-421.

22. Alvarez-Medina R, Le Dreau G, Ros M, Marti E. Hedgehog activation is required upstream of Wnt signalling to control neural progenitor proliferation. Development 2009; 136: 3301-3309.

23. Zhang N, Wei P, Gong A, Chiu WT, Lee HT, Colman H et al. FoxM1 promotes beta-catenin nuclear localization and controls Wnt target-gene expression and glioma tumorigenesis. Cancer Cell 2011; 20: 427-442.

24. Liu M, Dai B, Kang SH, Ban K, Huang FJ, Lang FF et al. FoxM1B is overexpressed in human glioblastomas and critically regulates the tumorigenicity of glioma cells. Cancer Res 2006; 66: 3593-3602.

25. Delpuech O, Griffiths B, East P, Essafi A, Lam EW, Burgering B et al. Induction of Mxi1-SR alpha by FOXO3a contributes to repression of Myc-dependent gene expression. Mol Cell Biol 2007; 27: 4917-4930.

26. Lin L, Hron JD, Peng SL. Regulation of NF-kappaB, Th activation, and autoinflammation by the forkhead transcription factor Foxo3a. Immunity 2004; 21: 203-213.

27. el-Deiry WS, Tokino T, Velculescu VE, Levy DB, Parsons R, Trent JM et al. WAF1, a potential mediator of p53 tumor suppression. Cell 1993; 75: 817-825.

28. Iotsova V, Stehelin D. Down-regulation of fibronectin gene expression by the p53 tumor suppressor protein. Cell Growth Differ 1996; 7: 629-634.

29. Su F, Pascal LE, Xiao W, Wang Z. Tumor suppressor U19/EAF2 regulates thrombospondin-1 expression via p53. Oncogene 2010; 29: 421-431. 
30. Zhou YH, Hu Y, Mayes D, Siegel E, Kim JG, Mathews MS et al. PAX6 suppression of glioma angiogenesis and the expression of vascular endothelial growth factor $\mathrm{A}$. J Neurooncol 2010; 96: 191-200.

31. Mayes DA, Hu Y, Teng Y, Siegel E, Wu X, Panda K et al. PAX6 suppresses the invasiveness of glioblastoma cells and the expression of the matrix metalloproteinase-2 gene. Cancer Res 2006; 66: 9809-9817.

32. Institute NCREMBRANDT home page. 2005 (20-11-2011); Available from: http:// rembrandt.nci.nih.gov.

33. Brennan C, Momota H, Hambardzumyan D, Ozawa T, Tandon A, Pedraza A et al. Glioblastoma subclasses can be defined by activity among signal transduction pathways and associated genomic alterations. PLoS One 2009; 4: e7752.

34. Cooper LA, Gutman DA, Long Q, Johnson BA, Cholleti SR, Kurc T et al. The proneural molecular signature is enriched in oligodendrogliomas and predicts improved survival among diffuse gliomas. PLoS One 2010; 5: e12548.

35. Sie M, Wagemakers M, Molema G, Mooij JJ, de Bont ES, den Dunnen WF. The angiopoietin 1/angiopoietin 2 balance as a prognostic marker in primary glioblastoma multiforme. J Neurosurg 2009; 110(1): 147-155.

36. Xu XL, Kapoun AM. Heterogeneous activation of the TGFbeta pathway in glioblastomas identified by gene expression-based classification using TGFbeta-responsive genes. J Transl Med 2009; 7: 12
37. Freije WA, Castro-Vargas FE, Fang Z, Horvath S, Cloughesy T, Liau LM et al. Gene expression profiling of gliomas strongly predicts survival. Cancer Res 2004; 64: 6503-6510.

38. Kimura T, Okada A, Yatabe T, Okubo M, Toyama Y, Noda M et al. RECK is up-regulated and involved in chondrocyte cloning in human osteoarthritic cartilage. Am J Pathol 2010; 176: 2858-2867.

39. Iversen PO, Drevon CA, Reseland JE. Prevention of leptin binding to its receptor suppresses rat leukemic cell growth by inhibiting angiogenesis. Blood 2002; 100: 4123-4128.

40. Crawford SE, Stellmach V, Murphy-Ullrich JE, Ribeiro SM, Lawler J, Hynes RO et al. Thrombospondin-1 is a major activator of TGF-beta1 in vivo. Cell 1998; 93: 1159-1170.

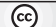

Cell Death and Disease is an open-access journal published by Nature Publishing Group. This work is licensed under the Creative Commons Attribution-NonCommercialShare Alike 3.0 Unported License. To view a copy of this license, visit http://creativecommons.org/licenses/by-nc-sa/3.0/

Supplementary Information accompanies the paper on Cell Death and Disease website (http://www.nature.com/cddis) 